\title{
EFECTO DE LA PODA DE FRUCTIFICACIÓN Y DEFOLIACIÓN EN EL PROCESO PRODUCTIVO DE CAMU CAMU EN LA REGIÓN UCAYALI-PERÚ
}

\author{
Carlos ABANTO-RODRÍGUEZ ${ }^{1}$, Mario PINEDO-PANDURO ${ }^{1}$, Ricardo BARDALES-LOZANO²， Edvan \\ ALVES-CHAGAS ${ }^{2}$ \\ 1 Instituto de Investigaciones de la Amazonía Peruana-IIAP. Programa en Manejo Integral del Bosque y Servicios \\ Ambientales (PROBOSQUES). Carretera Federico Basadre Km. 12.400, Pucallpa-Perú. E-mail: cabanto@iiap.org.pe, Av. \\ José A. Quiñones km. 2.5, distrito de San Juan Bautista, provincia de Maynas, departamento Loreto-Perú. E-mail: \\ mpinedo@iiap.org.pe, 2 Empresa Brasileira de pesquisa Agropecuária- Embrapa Roraima -Rodovia BR-174, Km 8 \\ Distrito Industrial Boa Vista, RR - Brasil - CEP 69301-970, E-mail: rbardaleslozano@yahoo.es, \\ edvan.chagas@embrapa.br.
}

\begin{abstract}
RESUMEN
Camu camu es una planta nativa de la Amazonía, que destaca por su alto contenido de vitamina C. Está en proceso de domesticación, por el cual se viene desarrollando modelos tecnológicos para lograr su desarrollo en suelos inundables y en tierra firme. El objetivo de este trabajo fue evaluar los efectos de la poda de fructificación combinados con diferentes tipos de defoliación, sobre el comportamiento productivo de plantas adultas de camu camu de 10 años de edad. El estudio fue conducido mediante un Diseño de Bloques Completos al Azar (DBCA) en esquema factorial 2Ax3B con 3 repeticiones y 16 plantas por unidad experimental, siendo el factor A: dos efectos de poda (Con poda y sin poda) y el factor B: tres tipos de defoliación (Manual, química y sin defoliación). Las variables evaluadas fueron: número y longitud de brotes, $\mathrm{n}^{\circ}$ de botones florales, $\mathrm{n}^{\circ}$ de frutos cuajados, $\mathrm{n}^{\circ}$ de frutos de cosecha y rendimiento de fruto en $\mathrm{t} \mathrm{ha}^{-1}$. Después de haber concluido el experimento se observó que el tratamiento Defoliación manual con poda fue estadísticamente superior a los demás tratamientos, destacándose en su mayor capacidad productiva de botones florales, frutos pequeños, frutos de cosecha y rendimiento en tha $\mathrm{a}^{-1}$ con un valor promedio de 11,135 ; 7,867; 1,862 y 19,7 respectivamente. Según los resultados obtenidos, la poda de fructificación combinada con la defoliación manual expresó mejor comportamiento productivo y mayores índices de fructificación.
\end{abstract}

PALABRAS CLAVE: Myrciaria dubia. Domesticación. Manejo agronómico. Rendimiento

\section{PRODUCTION OF CAMU CAMU WITH DIFFERENT TYPES OF PRUNING OF FRUITING AND DEFOLIATION ON THE UCAYALI REGION, PERU}

\section{ABSTRACT}

Camu camu is a plant native to the Amazon, known for its high content of vitamin C. Is in the process of domestication, for which it has been developing models to achieve technological development in floodplain soils and land. The aim of this study was to evaluate the effects of pruning fruiting combined with different defoliation on productive performance of adult plants of camu camu 10 years old. The study was conducted by a Design Randomized Complete Block (RCBD) in factorial scheme 2A x 3B with 3 replications and 16 plants per experimental unit, being the factor A two effects of pruning (with pruning and without pruning) and factor B three types of defoliation (Manual, without chemical defoliation). The variables evaluation were: number and shoot length $(\mathrm{cm}), \mathrm{n}^{\circ}$ of flower buds, $\mathrm{n}^{\circ}$ of fruit set, $\mathrm{n}^{\circ}$ fruit harvest and fruit yield in $\mathrm{tha}^{-1}$. Having completed the experiment it was observed that Defoliation manual treatment with pruning was statistically superior to the other treatments, highlighting the most productive capacity of flower buds, small fruits, fruit crop and yield in $\mathrm{tha}^{-1}$ with an average value of $11.135 ; 7.867 ; 1.862$ and 19.7 respectively. According to the results, pruning fruiting combined with manual defoliation expressed better growth performance and higher rates of fruiting.

KEYWORDS: Myrciaria dubia. Domestication. Agronomic management. Yield 


\section{INTRODUCCIÓN}

Una de las mayores tendencias del mundo moderno es a la orientación al consumo de productos orgánicos con alto valor nutritivo e vitamínico. Una alternativa es la fruticultura amazónica la cual es considerada como nicho potencial viable, que viene desarrollándose a través de la domesticación de nuevas especies y el desarrollo de modelos tecnológicos para lograr óptimos rendimientos (Abanto et al., 2014). Una de las especies nativas de la Amazonía de mayor importancia es el camu camu, el cual se destaca por ser una importante fuente de antioxidantes, debido a su alta concentración de ácido ascórbico (AA), conteniendo hasta 3,500 mg de AA $/ 100 \mathrm{~g}$ de pulpa, por lo que actualmente representa el recurso de la agrobiodiversidad amazónica con mayores perspectivas en el mercado nacional e internacional (Yuyama, 2011; Pinedo et al., 2010; Imán y Melchor, 2007).

En el año 1997 se instalaron 5274.43 has de camu camu en las Regiones de Loreto (3 997.13 has) y Ucayali (1 277.30 has) a nivel de pequeños productores, sin embargo en la actualidad solo sobreviven y están en producción aproximadamente 750 has en Loreto y 350 has en Ucayali (Pinedo et al., 2010). El $80 \%$ de las plantaciones fueron instaladas en espaciamientos de ( $3 \mathrm{~m} \times 3 \mathrm{~m} ; 3 \mathrm{~m} \times 2$ $\mathrm{m} ; 2 \mathrm{~m} \times 1,5 \mathrm{~m} ; 1 \mathrm{~m} \times 1,5 \mathrm{~m} ; 2 \mathrm{~m} \times 2 \mathrm{~m}$ ) entre líneas y entre plantas respectivamente (Penn, 2006; Picón y Acosta, 2001; Oliva, 2008). En relación a esto, Pinedo et al. (2001); Mathews y Yuyama (2010) indican que las densidades altas tienen la ventaja de producir mayores rendimientos en los primeros años, pero a medida que se incrementa la edad cronológica, se genera competencia progresiva por espacio, luminosidad y nutrientes, generando una disminución en la producción de fruta en más de 90\% por el surgimiento de problemas, como: el aumento de plagas y enfermedades, envejecimiento de ramas fruteras, mortandad de plantas, dificultad en la cosecha. También se crea sombreado excesivo, provocando la disminución del rendimiento, el tamaño, color y sólidos solubles del fruto e incluso induce a su caída (Flore y Layne, 1999).

En ese sentido para solucionar estos problemas y recuperar la producción es necesario implementar tecnologías de manejo agronómico que nos permita dar sostenibilidad al cultivo bajo un enfoque comercial a corto plazo, además los modelos tecnológicos servirán para manejar aproximadamente 8500 hectáreas nuevas de camu camu, instaladas en los últimos 5 años en Loreto y Ucayali. Dentro de las tecnologías que busca equilibrar la actividad vegetativa y reproductiva del árbol frutal, está la poda de fructificación o producción, que consiste en la eliminación ordenada de las estructuras aéreas de la planta, para fomentar el crecimiento de nuevos brotes, equilibrar la actividad vegetativa y reproductiva del arbusto, mediante una adecuada iluminación, para producir una eficiente fotosíntesis y aeración. El trabajo de poda también permitirá aplicar agrobio-insecticidas para disminuir las condiciones para el desarrollo de plagas y enfermedades (Navarro y Ramírez, 2010).

La poda de fructificación también busca mantener un alto nivel de regularidad y fruta de calidad todos los años, ya que se rejuvenece constantemente al árbol con lo cual se estimula el crecimiento y la producción de buenas estructuras fructíferas, igualmente se limita el volumen y altura de la planta controlando la forma de la copa, de modo que, los árboles que ya alcanzaron su tamaño deseado no continúen su expansión (García, 2008; Mataix, 1999).

Por otro lado la técnica de defoliación permite regularizar, uniformizar y mejorar la eficiencia productiva, a través del mantenimiento del equilibrio fisiológico de la planta (Imán y Melchor, 2007). A menudo las plantas presentan reposo prolongado o foliación retardada lo que causa el retraso en el crecimiento vegetativo y reproductivo (Erez, 1985). Al respecto Sánchez (2011), menciona que la remoción de hojas induce a una brotadura rápida de yemas laterales e incrementa la floración y además al defoliar, la producción y el almacenamiento de inhibidores son reducidos, estimulando el crecimiento de las yemas, siempre y cuando haya un nivel adecuado de sustancias promotoras del crecimiento.

Existen diferentes métodos de defoliacion entre ellos la defoliacion manual y la defoliacion química, la primera es una labor muy costosa y además gran parte de las ramas y yemas resultan dañadas, por lo que la defoliacion química ha cobrado gran importancia en zonas tropicales (Basak et al., 1973). Entre los productos químicos que han mostrado ser activos en el rompimiento de la latencia están: las sales minerales, como el sulfato de cobre, dimethipin y Dormex ${ }^{\circledR}$ (cianamida hidrogenada), este último ha obtenido resultados positivos en frutales en todo el mundo, actúa como un regulador de crecimiento estimulando el brotamiento de yemas en frutales tipo: manzano, peros, vid, olivo, durazno, etc. También funciona como defoliante en concentraciones medias a altas en zonas tropicales y subtropicales, además tiene la propiedad de degradarse fácilmente en úrea y amonio, actuando como fertilizante, sin dejar residuos en la cosecha ni en el suelo (Álvarez et al., 1992; Corzo, 1995; Erez, 1985). En este contexto, este estudio tuvo como 
objetivo evaluar los efectos de la poda de fructificación combinada con diferentes tipos de defoliación sobre el comportamiento productivo en plantas adultas de camu camu.

\section{MATERIAL Y MÉTODOS}

El experimento fue realizado durante los meses de octubre del 2010 hasta el mes de abril del 2011, en una parcela adulta de plantas de camu camu con 10 años de edad localizada en el caserío de San Pablo de Tushmo del Distrito de Yarinacocha Región Ucayali, con coordenadas UTM: 543964E; $9030116 \mathrm{~N}$ y 143 m.s.n.m. La precipitación y temperatura promedio registrada en el año 2010 fue de $1718 \mathrm{~mm}$ y de 27,57 ${ }^{\circ} \mathrm{C}$ y en el año 2011 fue de $1506 \mathrm{~mm}$ y una temperatura promedio de $28,50{ }^{\circ} \mathrm{C}$. El suelo del área experimental es un Entisol (suelos inundables), cuyo nivel de inundación promedio es de $1,8 \mathrm{~m}$, se caracteriza por presentar fertilidad media alta con materia orgánica de $1.36 \%$, Fósforo $7,48 \mathrm{mg} / \mathrm{kg}$, Potasio 58,65 mg/kg, pH de 5,22 y saturación de aluminio $12,89 \%$.

En el mes de octubre del año 2010, después de la cosecha se inició el experimento en plantas de camu camu de 10 años de edad, sembradas a un distanciamiento de $3 \mathrm{~m}$ entre líneas x $3 \mathrm{~m}$ ente plantas (1 111 plantas. ha ${ }^{-1}$ ). Se realizó podas de fructificación con diferentes tipos de defoliación, siguiendo las metodologías empleadas por Imán (2004); Imán y Melchor (2007); Inga (2009); Sánchez (2011) y Santarosa et al. (2010).

El experimento fue conducido mediante un diseño de bloques completamente al azar (DBCA), con arreglo factorial de $2 \mathrm{Ax} 3 \mathrm{~B}$ con 3 repeticiones y 16 plantas por unidad experimental, siendo el primer factor poda de fructificación con dos niveles: 1) con poda y 2) sin poda y el segundo factor defoliacion con 3 niveles: 1) sin defoliación, 2) defoliación manual y 3) defoliación química. Los tratamientos con poda se realizó a nivel del tercio superior de la planta (zona productiva) descartando el $15 \%$ de la copa, así mismo se eliminó ramas entrecruzadas mal posicionadas, excesivamente lignificadas, enfermas, secas e improductivas con la ayuda de serrucho cola de zorro, escaleras y tijeras de poda (Figuras 1ay 1b).

Para la defoliación química, se utilizó Cianamida hidrogenada en una concentración de $3 \%$, aplicada por aspersión en las primeras horas del día, utilizando una bomba aspersora de espalda de 20 litros, procurando una cobertura uniforme de todo el follaje (Quijada et al,, 1999; Imán y Melchor, 2007). Su aplicación se realizó durante el periodo de reposo del cultivo, para acelerar la caída de las hojas (Imán,
2004). Para controlar las plantas dañinas en la parcela se realizó cuatro deshierbos al año con la ayuda de una motoguadaña.

Los parámetros vegetativos evaluados fueron: número de brotes (NB) y longitud de brotes (LB) en $\mathrm{cm}$, para ello se dividió cada planta en cuatro cuadrantes norte, sur, este y oeste. En cada cuadrante se seleccionó una rama desde su origen en la cual se realizó la toma de datos según recomendaciones de Quijada et al.(1999).

Los parámetros productivos evaluados fueron: número de botones florales (NBF), número de frutos cuajados (NFC), número de frutos cosechados (NFC) y rendimiento de fruta en $t \mathrm{ha}^{-1}$ (RF). Los datos obtenidos fueron sometidos a análisis de varianza y los promedios fueron comparados mediante la prueba de Tukey al 5\% de probabilidad, todos los análisis fueron realizados con el programa estadístico SISVAR (Ferreira, 2010).

\section{RESULTADOS Y DISCUSIÓN \\ Número y longitud $(\mathrm{cm})$ de brotes}

Después de 25 días de haber instalado el experimento se realizó la evaluación del número y longitud de brotes $(\mathrm{cm})$. Según la prueba estadística de Tukey a un nivel de confianza superior a $95 \%$, se encontró interacción significativa entre el efecto de podas y los tipos de defoliación para los parámetros vegetativos número y longitud de brotes foliares. Para el número de brotes, el tratamiento defoliación química con poda presentó los mejores resultados, con un valor promedio de 142,08 brotes, seguido del tratamiento defoliación química sin poda con 110 brotes, siendo estadísticamente superiores al tratamiento sin defoliacion y sin poda el cual alcanzó un valor promedio de 29.42 brotes (Tabla 1). Este resultado coincide con Imán (2004), al trabajar con plantas adultas de camu camu, donde verificó que Cianamida hidrogenada al 3\% estimuló el desarrollo de brotes. De la misma forma Quijada et al. (1999), trabajando con defoliación en guayaba, demostraron que Cianamida hidrogenada incrementó significativamente la brotación de yemas independientemente de la poda.

El mejor resultado para la variable longitud de brotes $(\mathrm{cm})$ fue obtenido por el tratamiento defoliación manual con poda, con un valor de 28,20 $\mathrm{cm}$ en promedio (Tabla 1), el cual se comportó estadísticamente superior a los demás tratamientos. Con esto se demuestra que el crecimiento de los brotes es inversamente proporcional al número de brotes emitidos, tal como ocurrió con el tratamiento defoliación con Cianamida hidrogenada al 3\% con poda, quien obtuvo gran cantidad de brotes pero de menor longitud. 


\section{Botones florales y número de frutos cuajados}

Según la prueba estadística de Tukey $(\alpha<0,05)$ para botones florales y número de frutos cuajados (Tabla 2), fue verificado efectos simples para los tratamientos con poda y sin poda así como para los tipos de defoliación. Se registraron los mejores resultados en plantas que fueron tratadas con poda, obteniendo 8462.77 y 5805.28 botones florales y número de frutos cuajados respectivamente. Estos resultados coinciden con Quijada et al. (2009), quienes afirman que la poda de fructificación induce a la floración y formación de frutos en guayaba. En camu camu probablemente ocurrió lo mismo porque su floración es axilar y también porque el mayor porcentaje de flores se da en las ramas crecidas en el año y además en las que poseen entre 6 y $14 \mathrm{~mm}$ de diámetro (Pinedo et al., 2001). Del mismo modo Avilán et al. (1998), trabajando con podas de fructificación en mango, afirma que, los estudios realizados en relación con el comportamiento de los "flujos" de crecimiento de brotes, podados y sin podar, han determinado que los podados están "maduros" o aptos para florecer, cuando tienen de cinco a seis meses de edad, este hecho es lo que permite emplear la poda, ya que los nuevos brotes pueden dar origen a flores en el mismo año o ciclo de producción; por el contrario los no podados requieren entre diez a doce meses de edad para florecer.

En plantas sometidas a diferentes tipos de defoliación se encontró que el mejor tratamiento fue defoliación manual, con 8 802. 26 y 6375.92 para botones florales y número de frutos cuajados respectivamente, resultando estadísticamente superior a los tratamientos sin poda y sin defoliación (Tabla 2). En este sentido Campos et al. (2001), indican que, el uso de técnicas para estimular la brotación en árboles frutales puede ser una alternativa para adelantar, aumentar y uniformizar la apertura de yemas florales.

\section{Frutos de cosecha y Rendimiento de fruto en $\mathrm{t} \mathrm{ha}^{-1}$}

Según la prueba estadística de Tukey $(\alpha<0,05)$ para frutos de cosecha y rendimiento de fruto en $\mathrm{tha}^{-1}$ (Tabla 3), se encontró interacción significativa entre el efecto de podas y los tipos de defoliación. Para ambas variables el tratamiento defoliación manual con poda fue estadísticamente superior a los demás tratamientos, obteniendo un valor de 1862.25 frutos y 19,7 tha ${ }^{-1}$ respectivamente, en ese sentido Quijada et al. (2009) indica que la poda en los árboles frutales tropicales constituye una de las prácticas con mayores posibilidades de mejorar la producción a corto plazo. Esto pone en evidencia el efecto y la importancia de la poda de fructificación acompañada con la defoliación manual para camu camu, porque permitió el acceso a la plantación para realizar tratamientos sanitarios, la cosecha los raleos y también se mejoró la intercepción y distribución de la luz en las plantas, lo cual ayudó en la formación de flores, cuajado, desarrollo y calidad final de los frutos.

Según estos resultados el ensayo tuvo una duración de 206 días desde la instalación del experimento hasta la cosecha de frutos. En este sentido, Abanto (2011) trabajando con fertirrigación en plantas adultas de camu camu, constató que, una campaña agrícola de camu camu mediante un manejo agronómico de podas de fructificación y defoliación manual demoró 205 días desde la poda hasta la cosecha de frutos y además uniformizó y disminuyó el ciclo productivo de una campaña por año, a dos en 14 meses. De la misma manera Sánchez (2011) menciona que, después de haber aplicado poda de fructificación y defoliación manual a plantas adultas de camu camu, logró cosechar a los: 215; 203 y 206 días. También Imán (2004), reportó que una plantación de camu camu sometida a defoliación química con cianamida hidrogenada al 3\% tardó desde la defoliación hasta la cosecha de frutos 210 días. Del mismo modo Lopes (2008), trabajando con podas de fructificación en guayaba, verificó que el ciclo de producción desde la poda hasta la cosecha de frutos demoró aproximadamente 203 días.

\section{CONCLUSIONES}

Cianamida hidrogenada al 3\%, incrementó significativamente la brotación de yemas, independientemente de la poda pero no logró incrementar la longitud de los brotes.

El ciclo productivo desde la poda de fructificación y defoliación, hasta el inicio de la cosecha de frutos tardó un promedio de 206 días.

La poda más recomendada para la zona, es la poda de fructificación combinada con la defoliación manual debido a que presentó el mejor comportamiento productivo y mayores índices de fructificación. 


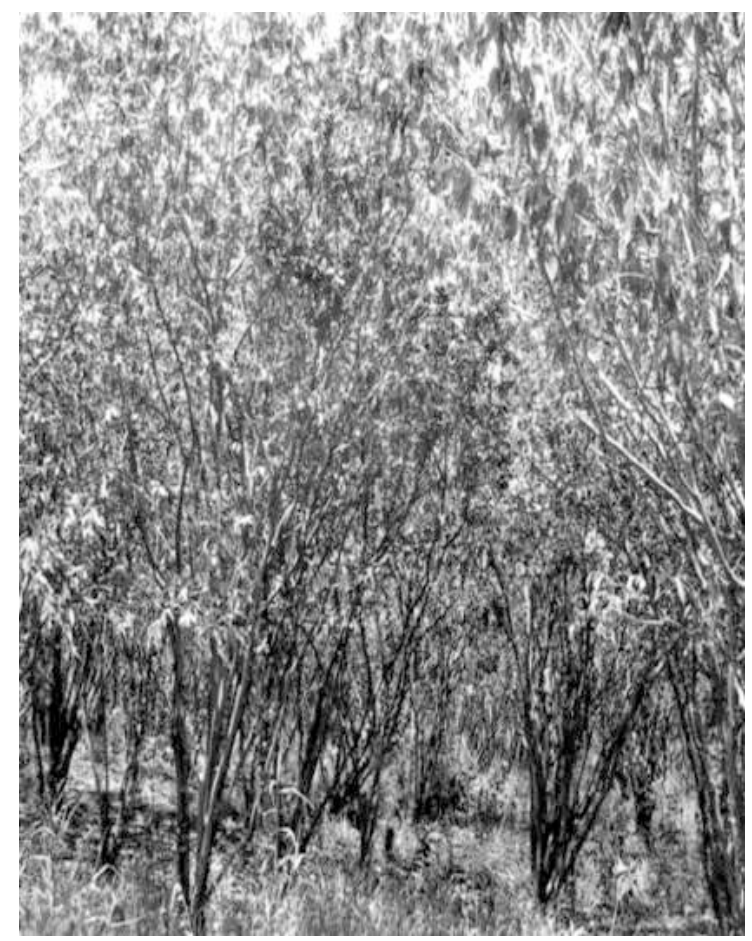

Figura 1a. Diagnóstico de la plantación de camu camu de 10 años de edad.

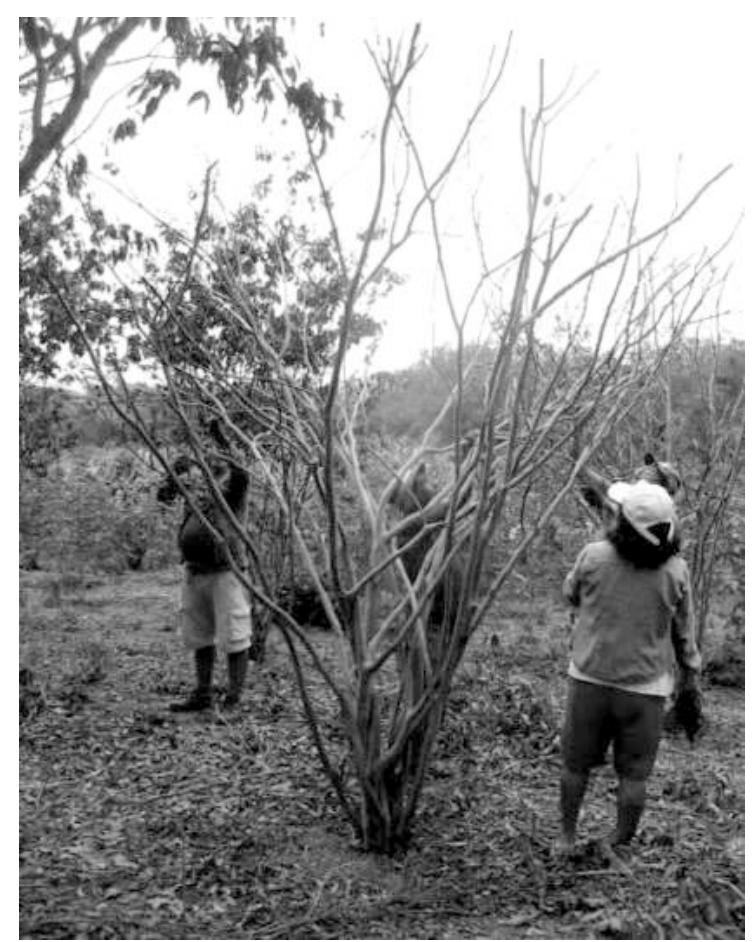

Figura 1b. Intervención de la plantación con métodos de podas de fructificación y defoliación. 
Tabla 1. Efecto de la poda de fructificación y defoliación en el número (NB) y longitud de brotes (LB)

\begin{tabular}{lrrrr}
\hline \multirow{2}{*}{ Tipo de Defoliación } & \multicolumn{3}{c}{ NB } & \multicolumn{3}{c}{ EB(cm) } \\
\cline { 2 - 5 } & Sin poda & Con poda & Sin poda & Con poda \\
\cline { 2 - 5 } & $29,42 \mathrm{bC}$ & $97,67 \mathrm{aB}$ & $12,10 \mathrm{aB}$ & $15,71 \mathrm{aB}$ \\
\hline Sin defoliación & $69,00 \mathrm{bB}$ & $81,33 \mathrm{aB}$ & $14,81 \mathrm{aB}$ & $28,20 \mathrm{bA}$ \\
Defoliación manual & $110,83 \mathrm{bA}$ & $142,0 \mathrm{aA}$ & $15,20 \mathrm{aB}$ & $11,42 \mathrm{aB}$ \\
\hline Defoliación química & 69,75 & 107,03 & 15,37 & 18,43 \\
\hline Promedio & &
\end{tabular}

Medias seguidas de letras minúsculas distintas en la fila, difieren entre sí por la prueba de Tukey $(\alpha<0,05)$.

Medias seguidas de letras mayúsculas distintas, en la columna, difieren entre sí por la prueba de Tukey $(\alpha<0,05)$.

Tabla 2. Efectos simples de la poda y defoliación en el número de botones florales (NBF) y número de frutos cuajados (NFC)

\begin{tabular}{ccc}
\hline \multirow{2}{*}{ Tipo de Defoliación } & NBF & NFC \\
\cline { 2 - 3 } & \multicolumn{2}{c}{ Efecto de Poda } \\
\hline Con Poda & 8462,77 a & $5805,28 \mathrm{a}$ \\
Sin Poda & $5775,10 \mathrm{~b}$ & $4036,83 \mathrm{~b}$ \\
\hline Promedio & \multicolumn{2}{c}{ Tipo de Defoliación } \\
\hline & 8921,05 \\
\hline Defoliación manual & $802,26 \mathrm{a}$ & $6375,92 \mathrm{a}$ \\
\hline Defoliación química & $6021,29 \mathrm{~b}$ & $4250,21 \mathrm{~b}$ \\
\hline Sin defoliación & $6533,25 \mathrm{~b}$ & $4137,04 \mathrm{~b}$ \\
\hline Promedio & 7118,93 & 4921,05 \\
\hline
\end{tabular}

Medias seguidas de letras distintas en la columna, difieren entre sí por la prueba de Tukey $(\alpha<0,05)$. 


\section{AGRADECIMIENTOS}

Agradecemos al Instituto de Investigaciones de la Amazonia Peruana-IIAP, por el apoyo financiero y al Sr. Fernando Murayari, productor de camu camu, por la cooperación para la realización de este trabajo.

\section{REVISIÓN BIBLIOGRÁFICA}

Abanto, C.; Chagas, E.A.; Sánchez-Choy, J.; Andrade, V.; Lozano, R.M.B.; Ríos, G.S. 2014. Capacidad de enraizamiento de plantas matrices promisorias de Myrciaria dubia (Kunth) Mc Vaugh en cámaras de subirrigación. Revista Ceres, 61(1): 134-140. doi: 10.1590/S0034737X2014000100018.

Abanto, C.; Oliva, C.; Domínguez, G.; Meza, A.; Alves, E. 2011. Fertirriego en la producción del camu camu (Myrciaria dubia HBK Mc Vaugh) en la estación experimental del IIAP, Ucayali, Perú. Scientia Agropecuaria 2(3): 157-167.

Avilán L.; Rodríguez, M.; Ruiz, J. 1998. Selección de cultivares de mango (Mangifera indica $\mathrm{L}$,) del Centro Nacional de Investigaciones Agropecuarias, Periodo 1952-1996. Proceedings of Interamericana Society for Tropical Horticulture, 42: 191-214.

Erez, A. 1985. Defoliation of deciduous fruit trees with magnesium chlorate and cyanamide. HortScience. 20 (3): 452-453.

Flore, J.A.; Layne, D.R. 1999. Photoassimilate production and distribution in cherry. HortScience 34(6): 1015-1019.

García, A.; Rodríguez. R. 2008. Poda de frutales. Estación Experimental Agropecuaria Alto Valle Centro Regional Patagonia Norte Instituto Nacional de Tecnología Agropecuaria. (http://www,inta,gov,ar/altovalle/actividad/desa rrollo/documentos/PodaFrutales,pdf). Acceso 01/08/2013.

Imán, C.S. 2004. ¿Cómo inducir una brotación uniforme de yemas axilares?.. Acceso 01/09/2011, Disponible en: http://www,inia, gob,pe/boletin/boletin0012/defoliante,htm.

Imán, C.S. 2004. ¿Cómo inducir una brotación uniforme de yemas axilares? (http://www,inia, gob,pe/boletin/boletin0012/defoliante,htm.). Acceso 01/09/2011.
Lopes, S.L.A.; Sales, M.C.; Melo, L.I.; Vagner, V.M.M.; Pagotto, R.C.; Dessaune, T.F. 2008. Fenologia da goiabeira 'paluma' sob diferentes sistemas de cultivos, épocas e intensidades de poda de frutificação. Bragantia, 67(3): 701-712. doi: 10.1590/S0006-87052008000300019.

Mataix, E.; Villarrubia, D. 1999. Poda de frutales. Generalitat Valenciana, Valencia.104pp. (http://www,ivia,es/sdta/pdf/libros/n45,pdf). Acceso 12/07/2013.

Mathews, D.P.; Yuyama, K. 2010. Comprimento de estacas de camu-camu com ácido indolbutírico para a produção de mudas. Revista Brasileira de Fruticultura, 32(2): 522-526. doi: 10.1590/S0100-29452010005000066.

Navarro, O. M.; Ramírez, I.J. 2010. Obtención de frutos de calidad en manzanas. Revista Frutícola, 2: 4-9.

Penn, J.W. 2006. The cultivation of camu camu (Myrciaria dubia): A tree planting programme in the Peruvian Amazon. Forests, Trees and Livelihoods, 16(1): 85-101. doi: 10.1080/14728028.2006.9752547.

Quijada, O.; Ramírez, R.; Castellano, G.; Camacho, R.; Burgos, M. 2009. Tipos de poda y producción en guayabo (Psidium guajava L,) en el municipio Baralt, estado Zulia, Venezuela. Revista Científica UDO Agrícola, 9(2): 304-311. (http://www.bioline.org.br/request?cg09039). Acceso 02/09/2013.

Quijada, O.; Araujo, F.; Corzo, P. 1999. Efecto de la poda y la cianamida hidrogenada sobre la brotación, fructificación, producción y calidad de frutos del guayabo (Psidium guajava $\mathrm{L}$,) en el municipio Mara del estado Zulia, Revista de la Facultad de Agronomía (LUZ), 16: 276-290.

Santarosa, E.; Koller, O.C.; Petry, H.B.; Casamali. B. 2010. Frequência e intensidade de poda em pomar jovem de laranjeiras 'Valência' sob manejo orgânico. Ciência Rural, 40(10): 20812085. doi: 10.1590/S0103-84782010005000167

Recibido: 12/01/2014

Aceptado para publicación: 22/04/2014 
